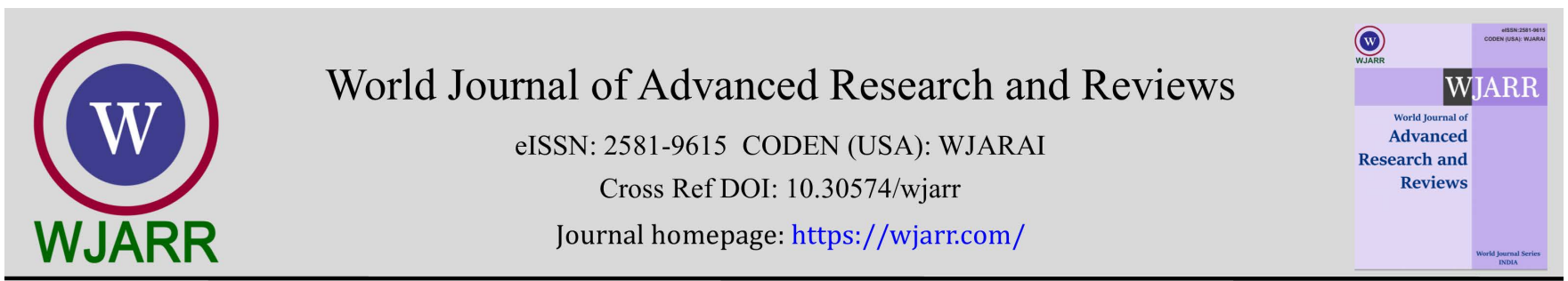

(RESEARCH ARTiClE)

Check for updates

\title{
Analysis of microflora in dentinal tubule by denaturing gradient gel electrophoresis
}

\author{
Norimasa Tsuji *, Sadaomi Sugimoto, Hitomi Nakama and Hiroshi Maeda \\ Department of Endodontics, School of Dentistry, Osaka Dental University 8-1 Kuzuhahanazonocho Hirakata, Osaka 573- \\ 1121, Japan.
}

World Journal of Advanced Research and Reviews, 2021, 11(02), 085-092

Publication history: Received on 30 June 2021; revised on 04 August 2021; accepted on 06 August 2021

Article DOI: https://doi.org/10.30574/wjarr.2021.11.2.0362

\begin{abstract}
This study was performed to apply denaturing gradient gel electrophoresis to microbiological examination of endodontic infections. The method was used to assess the bacterial communities in dentinal tubules. Samples were collected using \#15, \#35, and \#60 K-type files from five infected root canals during treatment. Deoxyribonucleic acid(DNA) was extracted from the samples, and 16S ribosomal DNA was amplified by PCR using universal primers. The polymerase chain reaction(PCR) products were separated in the denaturing gel and band patterns were compared between the deep (\#60 file) and superficial layers (\#15 file) of the dentin. The major bands were then excised and DNA fragments in the gel were cloned and sequenced. The sequence data were subjected to BLAST search in the GenBank database for determination of bacterial species or closest relatives. In three root canals, similar band patterns were observed in both superficial and deep layers of the dentin, while several specific bands remained in the deep layer in two roots. The bacteria isolated from the deep layers were Porphyromonas gingivalis, Olsenella profuse, Atopobium rimae, and Prevotella, Flexistipes, Firmicutes, Peptostreptococcus, Dialister, and Eubacterium spp. Unlike previous studies, gram-negative anaerobic rods were isolated from the deep layers. Clone library analysis was simultaneously performed and similar results were obtained. The method utilized here will be useful for microbiological examination of endodontic infections. In addition, although it is still unknown whether they were viable, this study demonstrated the presence of gram-negative rods in dentinal tubules.
\end{abstract}

Keywords: Denaturing gradient gel electrophoresis (DGGE); 16S ribosomal ribonucleic acid(RNA); Dentinal tubule; Endodontic infection

\section{Introduction}

Advances in molecular biological techniques have enabled the detection of uncultivable bacteria in root canals and revealed that bacterial community structure is more diverse than previously thought based on culture methods [1, 2, $3]$. The dentinal tubule microflora has been characterized using culture-based techniques $[4,5,6]$. Although wide ranges of bacteria, including gram-negative anaerobes, were found in root canals, relatively limited bacterial species were isolated from the deep dentinal tubules. Gram-positive obligate anaerobes, mainly strains belonging to the genera, Lactobacillus and Streptococcus, have been reported to be predominant in the deeper dentinal tubule. These bacteria were isolated from areas $0.5-2.0 \mathrm{~mm}$ from the canal-dentin boundary [7]. Gram-negative anaerobes, such as Porphyromonas, Fusobacterium, and Prevotella species, have been suggested to be associated with acute symptoms of apical lesions. However, they have not been isolated from the deep dentinal tubule [7]. Therefore, more sensitive analysis is required to determine the true diversity of microflora in the dentinal tubule, especially deeper within the tubule.

\footnotetext{
${ }^{*}$ Corresponding author: Norimasa Tsuji

Department of Endodontics, School of Dentistry, Osaka Dental University 8-1 Kuzuhahanazonocho Hirakata, Osaka 573-1121, Japan. Copyright (C) 2021 Author(s) retain the copyright of this article. This article is published under the terms of the Creative Commons Attribution Liscense 4.0.
} 
PCR is now widely used for microbiological diagnosis in various infectious diseases. However, the method still has some limitations. Even for polymicrobial communities, such as those in the root canal, PCR targets have to be selected, and therefore the bacterial diversity of the whole community remains unclear. A new approach for polymicrobial ecology is based on $16 \mathrm{~S}$ ribosomal DNA (16S rDNA) information obtained by clone library analysis [2, 3]. The method appears to be very useful for determining the composition of bacterial communities. However, the analysis requires a number of laborious cloning and sequencing tasks.

Denaturing gradient gel electrophoresis (DGGE) was originally described for mutation analysis [8, 9]. Recently, this molecular technique has been shown to be useful in microbial ecology [10]. As sequence-specific separation of 16S rDNA and further sequencing or hybridization analysis are possible, DGGE has become a powerful tool to examine bacterial diversity in various natural habitats, such as marine or soil environments [11]. This method has also been employed in the medical and dental fields for analysis of polymicrobial communities in humans $[12,13,14]$.

Bacteria in the root canal play essential roles in apical periodontitis, and the remaining bacteria in dentinal tubules may be associated with persistent endodontic infections [16]. Understanding the microflora in the root canal and dentinal tubule is indispensable for the improvement of treatment strategies for endodontic infection. In the present study, the DGGE method was applied to microbiological examination of endodontic infections. The profiles of the bacterial communities in both root canals and dentinal tubules were simultaneously analyzed using DGGE.

\section{Material and methods}

\subsection{Collection of endodontic samples}

Samples were collected from five teeth from five patients. Single-rooted teeth with periapical radiolucency $(2.0-3.0$ $\mathrm{mm}$ in diameter) and without severe periodontal disease (periodontal pockets $<4 \mathrm{~mm}$ ) were selected for the study. None of the root canals had been treated previously with endodontic therapy. They had carious lesions and necrotic pulp. Four teeth were asymptomatic except for slightly tenderness to percussion. Dental fistula was observed in the remaining one case. The diameter of all canals near the root apex was almost the same as that of a \#15 K-type file (Dentsply/Maillefer).

The selected teeth were isolated with a rubber dam, carious lesions were removed, and cleansed with $3 \%$ hydrogen peroxide. Complete access preparations were made, and the third of the root canal from the orifice was enlarged into a funnel-shape using a Pesso reamer (Pierce). Samples were collected initially with a \#15 K-type file. The file was introduced to a level approximately $0.5 \mathrm{~mm}$ short of the tooth apex by monitoring with an electric apex locator (Morita), and the necrotic pulp and dentin powder attached to the file were suspended in $1 \mathrm{ml}$ of PBS (-) (Gibco-BRL). Canal preparation was performed using K-type files and the canal was irrigated with $3 \%$ hydrogen peroxide and $2.5 \%$ sodium hypochlorite between each file size. Second and third samples were collected by \#35 and \#60 K-type files in the same way. The Ethics Committee of Osaka Dental University approved the protocol of this study (approval number: 110972), and all patients provided written informed consent to participate in the study.

\subsection{DNA extraction}

Total bacterial DNAs were extracted from the clinical samples using InstaGene Matrix (Bio-Rad) according to the manufacturer's instructions. Briefly, bacterial samples in $1 \mathrm{ml}$ of PBS (-) (Gibco-BRL) were pelleted and resuspended in $100 \mu \mathrm{l}$ of InstaGene Matrix (Bio-Rad). The suspension was incubated at $56^{\circ} \mathrm{C}$ for $30 \mathrm{~min}$ and then $100^{\circ} \mathrm{C}$ for $8 \mathrm{~min}$. After incubation, the suspension was centrifuged and $5 \mu \mathrm{l}$ of the resulting supernatant was used for PCR prior to DGGE.

\subsection{PCR-DGGE analysis}

The 16S rDNA fragments (nucleotide positions 341 - 926 in the Escherichia coli sequence) were amplified with two conserved primers. A forward primer with a 40-bp GC clamp added to its 5'-end (5'cgcccgccgcgccccgcgcccgtcccgccgcccccgcccg-cctacgggaggcagcag-3') and reverse primer ( $5^{\prime}$-ccgtcaattcctttRagttt- $\left.3^{\prime}\right)$ were prepared for PCR. Amplification was carried out using a High-Fidelity PCR Master Kit (Roche Molecular Biochemicals) in $50-\mu \mathrm{l}$ reactions. A touchdown procedure $\left(65^{\circ} \mathrm{C}-55^{\circ} \mathrm{C}\right.$ annealing temperature) was adopted for thermocycling [17].

The DCode Universal Mutation Detection System (Bio-Rad) was used for gel electrophoresis. The PCR products (20 $\mu \mathrm{l})$ were loaded onto an $8 \%(\mathrm{w} / \mathrm{v})$ polyacrylamide gel containing a linear gradient ranging from $20 \%$ to $50 \%$ denaturant (7 M urea and $40 \%$ formamide). The gel was run for 3 hours at $200 \mathrm{~V}$ in $1 \times$ TAE buffer $(0.04 \mathrm{M}$ Tris-acetate/1 mM EDTA, $\mathrm{pH} 8.5$ ) at a constant temperature of $60^{\circ} \mathrm{C}$. After electrophoresis, the gel was stained with ethidium bromide, and the amplified DNA bands were visualized with a Fluor-S Multilmager (Bio-Rad). 


\subsection{Sequence analysis of DGGE bands}

The major bands on the denaturing gel were excised, purified, and cloned into the PCR 2.1 vector (Invitrogen). The insert DNA was sequenced using an ABI Prism BigDye terminator cycle sequencing ready reaction kit (Perkin Elmer) and an automated DNA sequencer (ABI PRISM 377; Perkin Elmer). The sequence data were subjected to BLAST search in the GenBank database.

\subsection{Clone library analysis}

Microflora in one of the five root canals was simultaneously evaluated by clone library analysis. For construction of the library, 16S rDNA was amplified using the universal primers 27F and 1525R. The amplified 16S rDNA fragments were ligated into the PCR 2.1 vector, and E. coli XL1-blue was transformed with the ligation mixtures. The transformants were randomly picked up and plasmids were isolated. Sequencing and BLAST search were performed as described above.

\section{Results}

Prior to DGGE analysis, PCR products were subjected to electrophoresis in 1.5\% agarose gels. A single band was detected from each sample at the expected size (585 bp, data not shown). The DGGE profiles of the amplified 16S rDNA are shown in Figure 1.

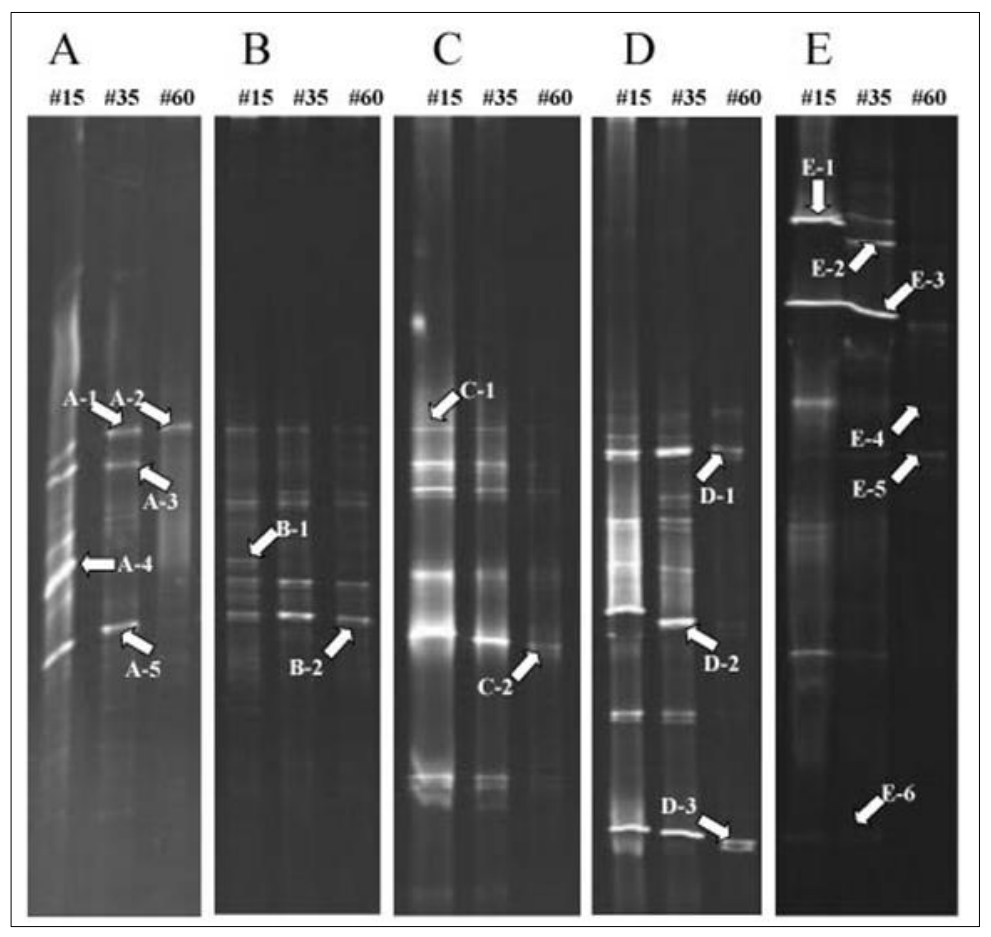

Figure 1 DGGE profiles of amplified 16S rDNA

Samples were collected from five root canals (A - E) at different distances from the canal-dentin boundary during root canal treatment with \#15, \#35, and \#60 K-type files, and were subjected to DGGE analysis as described in the Materials and methods. Eighteen intense bands indicated by arrows were cloned and sequenced. The bacterial species identified from the sequence data are shown in Table 1.

The multiple 16S rDNA fragments amplified by PCR were separated according to sequence specificity. Distinct band patterns were observed among the different root canals, although several bands were common to more than two canals (A-1 and C-1; A-3 and D-1; A-5, B-2 and D-2). From the surface to the deep dentin layer, the DGGE profile did not change dramatically in root canals $\mathrm{B}, \mathrm{C}$, and E. However, in root canals A and D, several selected bands remained in deep dentin layer (bands A-2, D-1, and D-3).

To determine the dominant species in each sample, selected major bands were cloned and sequenced. The selected bands are indicated by arrows in Figure 1 (numbered A - G). Four clones were isolated and sequenced from each band and the nucleotide sequences $(250-300 \mathrm{bp})$ were compared with $16 \mathrm{~S}$ rDNA in the database. All sequence data of the 
clones were closely affiliated with existing sequence data in GenBank with similarity values of $98 \%-100 \%$. The identified bacterial species are shown in Table 1.

Table 1 Identified bacteria from DGGE bands

\begin{tabular}{|c|c|c|c|}
\hline $\begin{array}{c}\text { Sample } \\
\text { (DGGE bands }{ }^{\mathrm{a}} \text { ) }\end{array}$ & Phylotype/species & $\begin{array}{l}\text { Accession } \\
\text { no. }\end{array}$ & $\begin{array}{l}\text { No. of isolated clones } \\
\text { (Four clones from each band) }\end{array}$ \\
\hline$A-1$ & Porphyromonas gingivalis & AF414809 & 4 \\
\hline$A-2$ & Porphyromonas gingivalis & AF414809 & 4 \\
\hline$A-3$ & Firmicutes sp. oral clone & AF287778 & 4 \\
\hline$A-4$ & Lautropia sp. oral clone & AY134902 & 4 \\
\hline$A-5$ & Flexistipes-like sp. oral clone & AY005444 & 4 \\
\hline \multirow[t]{2}{*}{ B-1 } & Fusobacterium sp. oral clone & AF287805 & 1 \\
\hline & Fusobacterium nucleatum & AF543300 & 3 \\
\hline $\mathrm{B}-2$ & Flexistipes-like sp. oral clone & AY005444 & 4 \\
\hline $\mathrm{C}-1$ & Porphyromonas gingivalis & AF414809 & 4 \\
\hline $\mathrm{C}-2$ & $\begin{array}{l}\text { Uncultured fiber-attaching rumen } \\
\text { bacterium }\end{array}$ & $\mathrm{AB} 045742$ & 4 \\
\hline D-1 & Firmicutes sp. oral clone & AF287778 & 4 \\
\hline $\mathrm{D}-2$ & Flexistipes-like sp. oral clone & AY005444 & 4 \\
\hline $\mathrm{D}-3$ & Olsenella profuse & AF292374 & 4 \\
\hline \multirow[t]{2}{*}{ E-1 } & Lachnospiraceae oral clone & AF481220 & 3 \\
\hline & Staphylococcus sp. B3117 & AF322002 & 1 \\
\hline \multirow[t]{2}{*}{ E-2 } & Alpha Proteobacterium sj22 & AF530921 & 2 \\
\hline & $\begin{array}{l}\text { Propionibacterineae bacterium clone } \\
\text { RH-B24N }\end{array}$ & AF513962 & 2 \\
\hline \multirow[t]{3}{*}{ E-3 } & Peptostreptococcus sp. oral clone & AF287763 & 2 \\
\hline & Dialister sp. oral isolate & AF481209 & 1 \\
\hline & Atopobium rimae & AF292371 & 1 \\
\hline \multirow[t]{2}{*}{ E-4 } & Prevotella buccae & L16477 & 2 \\
\hline & Prevotella nigrescens & AF414844 & 2 \\
\hline E-5 & Eubacterium sp. oral clone & AF287764 & 4 \\
\hline E-6 & Olsenella profuse & AF292374 & 4 \\
\hline
\end{tabular}

Porphyromonas gingivalis, Olsenella profuse, Atopobium rimae, and Prevotella, Flexistipes, Firmicutes, Peptostreptococcus, Dialister, and Eubacterium spp. were identified in the deep layers. Among them, P. gingivalis, Firmicutes, Flexistipes, and Olsenella spp. were identified from multiple root canals. Band A-1 indicated that P. gingivalis was still dominant in the deep layer (A-2), while other dominant bands in sample A disappeared during endodontic therapy. Similarly, D-1 indicating Firmicutes sp. and D-3 indicating Olsenella were selectively dominant in the deep dentin layer.

Clone library analysis was performed using the samples from root canal E. Thirty clones were isolated and sequenced in each layer, and were identified to the genus level by comparison with the DNA database. The results are shown in Table 2. 
Table 2 Isolated bacteria by clone library analysis (sample E)

\begin{tabular}{|c|c|c|c|}
\hline \multirow{2}{*}{ Bacterial species } & \multicolumn{3}{|c|}{ aNumber of clones } \\
\hline & \#15 & \#35 & \#60 \\
\hline Peptostreptococcus sp. & 8 & 10 & 8 \\
\hline Dialister sp. & 6 & 10 & 7 \\
\hline Lachnospiraceae sp. & 4 & 4 & 1 \\
\hline Firmicutes sp. & 3 & 4 & 1 \\
\hline Atopobium sp. & 2 & 2 & 3 \\
\hline Olsenella sp. & 2 & 2 & 2 \\
\hline Methylobacterium sp. & 1 & 1 & 4 \\
\hline Eubacterium sp. & 2 & 2 & 2 \\
\hline Propionibacterium sp. & 1 & 0 & 1 \\
\hline Unknown & 1 & 2 & 1 \\
\hline
\end{tabular}

Clones with sequences identity < 98\% were found in some cases, and are shown as "unknown." The bacterial community structures of different dentin layers were almost the same and were dominated by Dialister and Peptostreptococcus spp. The dominant bacteria isolated by clone library analysis and DGGE method were almost identical.

\section{Discussion}

As wide ranges of bacterial species have been isolated from root canals [3, 4, 18, 19], DGGE showed multiple bands, and the band patterns were different between the sampled teeth (Fig. 1). The results indicated the presence of complex polymicrobial communities in root canals, and that the composition of microorganisms was different in each root canal or patient. On the other hand, several major bands were common among different samples. The relative quantity of DNA in the individual bands generally suggested numerical dominance. Bacterial species dominating the multiple root canals, such as P. gingivalis, Firmicutes, and Flexistipes, may be adapted to the environment of the root canal. Consistent with previous reports $[3,18,19]$, the Firmicutes phylum (Table 1) was dominant in most cases. In contrast, the microflorae of the deep dentin layers were inconsistent with previous reports. Using culture-based techniques, the microflorae of the deep dentin layers were reported to be dominated by gram-positive anaerobes, such as lactobacilli and streptococci [7]. It has also been suggested that gram-negative anaerobic rods are not able to invade dentinal tubules [20]. However, in this study, gram-negative rods, such as Porphyromonas and Prevotella, were found to be dominant in the deep layers of the dentin. Dialister, which is a current topic of interest in periodontal disease [21, 22, 23, 24], was also identified in the deep layer. Supporting our results, these bacteria have been suggested to have the ability to invade the dentinal tubule [25], as they can penetrate into the dentin wall from the periodontal ligament side [26]. The gram-negative anaerobes are thought to co-invade with streptococci or other facultative anaerobes [26]. It should also be noted that only several major bands in each sample were sequenced in this study and many unidentified bands remained in the deep dentin layer, which may include lactobacilli or streptococci. The bacterial community structure in dentinal tubules appeared to be more diverse than previously thought based on culture methods, and the present study using DGGE suggested that gram-negative anaerobes can invade into the dentinal tubule from the pulpal side. Limitations of culturebased techniques may have affected the results of previous studies, leading to the underestimation of bacterial diversity.

We compared the DGGE profiles between the superficial (\#15 file) and deep dentin layers (\#60 file) to investigate whether specific bacteria were selected to penetrate the dentin wall from among the whole bacterial community of the original root canal. In root canal A (Fig. 1), P. gingivalis bands (A-1, A-2) were observed throughout the therapy with similar intensity, whereas other bands disappeared in the deep layer. Similarly, Firmicutes (D-1) and Olsenella (D-3) were selected in root canal D. In contrast, the band pattern did not change from surface to deep layers in other root canals although bacterial numbers (density of each band) gradually decreased. Interestingly, $P$. gingivalis of root canal A was specifically selected from among the whole bacterial community and invaded into the deep layer, while the population of $P$. gingivalis of root canal $C$ gradually decreased from the surface to the deep layer. Specific bacteria were selected and invaded dentinal tubules in two cases. However, the microflorae in dentinal tubules were similar to those 
of the original root canals in the other three cases. We speculated that bacterial cell-cell interactions among the different strains may be important for invasion. Further detailed studies of the microbial community structure in larger samples are required. Sample E from a tooth with fistula demonstrated a distinct band pattern from the others with bands at high molecular weight positions. Investigation of the relation between the clinical aspects and DGGE profile will also be necessary.

Clone library analysis was performed to assess the utility of DGGE. The identified bacteria were mostly common between the two methods. The results demonstrated that DGGE could be a powerful tool for evaluation of polymicrobial community structures in cases of endodontic infection. Clone library analysis is a reliable technique, but is not practical for routine examinations as it requires numerous sequencing steps. Therefore, DGGE may have a methodological advantage for clinical examinations.

The present study demonstrated the great potential of DGGE for determining the polymicrobial community structure in cases of endodontic infection. However, several minor limitations should also be discussed. For example, the $16 \mathrm{~S}$ rDNA band in DGGE sometimes reflects the presence of multiple microbial species [10]. In fact, several species were identified from single bands in the DGGE profile of sample E. It may be difficult to separate the 16S rDNA bands completely when closely related species are present in large numbers. To resolve these problems, primer design or electrophoresis conditions should be optimized. Another problem is related to the viability of identified bacteria. As genomic DNA could be recovered from non-viable microorganisms, the method used in this study was not able to assess the viability of the isolates. Nevertheless, the presence of DNA in the deeper tubule would suggest active invasion of these bacteria in the clinical course of endodontic infections.

DGGE was able to express the complicated microflora visually with its band patterns, and sequencing of the major bands revealed the dominant bacteria in the dentinal tubules. The presence of gram-negative anaerobic rods in the dentinal tubule may require more attention for disinfection during endodontic therapy.

\section{Conclusion}

The DGGE method and clone library analysis were applied for microbiological examination of infected root canals, and both methods revealed the presence of gram-negative anaerobic rods in the deep dentin layers. The DGGE method has potential to become a powerful tool for analyzing the changes in the microflora in root canals through endodontic therapy.

\section{Compliance with ethical standards}

\section{Acknowledgments}

This study was supported in part by a Grant-in-Aid for Scientific Research (Grant number: 18K09613) from Japan Society for the Promotion of Science.

\section{Disclosure of conflict of interest}

The authors have no conflicts of interest to clear.

\section{References}

[1] Jung IY, Choi BK, Kum KY, Roh BD, Lee SJ, Lee CY, Park DS. Molecular epidemiology and association of putative pathogens in root canal infection. Journal of Endodontics. 2000; 26: 599-604.

[2] Rolph HJ, Lennon A, Riggio MP, Saunders WP, MacKenzie D, Coldero L, et al. Molecular identification of microorganisms from endodontic infections. Journal of Clinical Microbiology. 2001; 39: 3282-9.

[3] Munson MA, Pitt-Ford T, Chong B, Weightman A, Wade WG. Molecular and cultural analysis of the microflora associated with endodontic infections. Journal of Dental Research. 2002; 81: 761-6.

[4] Sakko M, Tjäderhane L, Rautemaa-Richardson R. Microbiology of Root Canal Infections. Primary Dental Journal. 2016; 5(2): 84-9. 
[5] Bumb SS, Bhaskar DJ, Agali CR, Punia H, Gupta V, Singh V, Kadtane S, Chandra S. Assessment of photodynamic therapy (PDT) in disinfection of deeper dentinal tubules in a root canal system: an in vitro study. Journal of Clinical and Diagnostic Research. 2014; 8(11): ZC67-71.

[6] Peters LB, Wesselink PR, Bujis JF, van Winkelhoff AJ. Viable bacteria in root dentinal tubules of teeth with apical periodontitis. Journal of Endodontics. 2001; 27: 76-81.

[7] Ando N, Hoshino E. Predominant obligate anaerobes invading the deep layers of root canal dentin. International Endodontic Journal. 1990; 23: 20-7.

[8] Fischer SG, Lerman LS. DNA fragments differing by single base-pair substitutions are separated in denaturing gradient gels: correspondence with melting theory. Proceedings of the National Academy of Sciences of the United States of America. 1983; 80: 1579-83.

[9] Myers RM, Maniatis T, Lerman LS. Detection and localization of single base changes by denaturing gradient gel electrophoresis. Methods in Enzymology. 1987; 155: 501-27.

[10] Muyzer G, De Waal EC, Uitterlinden AG. Profiling of complex microbial populations by denaturing gradient gel electrophoresis analysis of polymerase chain reaction-amplified genes coding for 16S rRNA. Applied and Environmental Microbiology. 1993; 59: 695-700.

[11] Muyzer G, Smalla K. Application of denaturing gradient gel electrophoresis (DGGE) and temperature gradient gel electrophoresis (TGGE) in microbial ecology. Antonie van Leeuwenhoek. 1998; 73: 127-41.

[12] Raviraj GA, Bhat KG, Kugaji MS, Kumbar VM, Hooli A. Study of microbial diversity in saliva and plaque samples from caries-free and caries-affected children using denaturing gradient gel electrophoresis. Journal of the Indian Society of Pedodontics and Preventive Dentistry. 2018; 36(4): 396-401.

[13] Schabereiter-Gurtner C, Maca S, Rolleke S, Nigl K, Lukas J, Hirschl A, et al. 16S rDNA-based identification of bacteria from conjunctival swabs by PCR and DGGE fingerprinting. Investigative Ophthalmology and Visual Science. 2001; 42: 1164-71.

[14] Fujimoto C, Maeda H, Kokeguchi S, Takashiba S, Nishimura F, Arai H, et al. Application of denaturing gradient gel electrophoresis (DGGE) to the analysis of microbial communities of subgingival plaque. Journal of Periodontal Research. 2003; 38(4): 440-5.

[15] Zijinge V, Harmsen HJM, Kleinfelder JW, van der Rest ME, Degener JE, Welling GW. Denaturing gradient gel electrophoresis to study bacterial community structure in pockets of periodontitis patients. Oral Microbiology and Immunology. 2003; 18: 59-65.

[16] Bystrom A, Happonen RP, Sjogren U, Sundqvist G. Healing of periapical lesions of pulpless teeth after endodontic treatment with controlled asepsis. Endodontics and Dental Traumatology. 1987; 3: 58-63.

[17] Don RH, Cox PT, Wainwright BJ, Baker K, Mattick JS. Touchdown PCR to circumvent spurious priming during gene amplification. Nucleic Acids Research. 1991; 19: 4008.

[18] Sundqvist G. Ecology of the root canal flora. Journal of Endodontics. 1992; 18: 427-30.

[19] Sundqvist G. Taxonomy, ecology, and pathogenicity of the root canal flora. Oral Surgery, Oral Medicine, and Oral Pathology. 1994; 78: 522-30.

[20] Akapta ES, Blechman H. Bacterial invasion of pulpal dentin wall in vitro. Journal of Dental Research. 1982; 61: 435-8.

[21] Mohammed SR, Anand N, Chandrasekaran SC, Mahalakshmi K, Padmavathy K. Evaluation of periodontal status and detection of Dialister pneumosintes in cerebral palsy individuals: a case-control study. Indian Journal of Dental Research. 2018; 29(6): 768-72.

[22] Siqueira JF Jr, Rôças IN. Microbiology and treatment of acute apical abscesses. Clinical Microbiology Reviews. 2013; 26(2): 255-73.

[23] Ghayoumi N, Chen C, Slots J. Dialister pneumosintes, a new putative periodontal pathogen. Journal of Periodontal Research. 2002; 37: 75-8.

[24] Rousee JM, Bermond D, Piemont Y, Tournoud C, Heller R, Kehrli P, Harlay ML, Monteil H, Jaulhac B. Dialister pneumosintes associated with human brain abscesses. Journal of Clinical Microbiology. 2002; 40: 3871-3. 
[25] Love RM, McMillan MD, Park Y, Jenkinson HF. Coinvasion of dentinal tubules by Porphyromonas gingivalis and Streptococcus gordonii depends upon specificity of streptococcal antigen I/II adhesin. Infection and Immunity. 2000; 68: 1359-65.

[26] Adriaens PA, de Boever JA, Loesche WJ. Bacterial invasion in root cementum and radicular dentin of periodontally diseased teeth in humans. Journal of Periodontology. 1988; 59: 222-30. 